\title{
Correction to: Transcriptomic analysis of hepatic responses to testosterone deficiency in miniature pigs fed a high- cholesterol diet
}

Zhaowei Cai ${ }^{1}$, Xiaoling Jiang ${ }^{2}$, Yongming Pan ${ }^{1}$, Liang Chen ${ }^{1}$, Lifan Zhang ${ }^{3}$, Keyan Zhu' ${ }^{1}$ Yueqin Cai ${ }^{1}$, Yun Ling ${ }^{1}$, Fangming Chen ${ }^{1}$, Xiaoping $\mathrm{Xu}^{1}$ and Minli Chen ${ }^{1 *}$

\section{Correction to: BMC Genomics}

$$
\text { https://doi.org/10.1186/s12864-015-1283-0 }
$$

Following the publication of the original article [1], it was reported that the accession number given in the 'Data accessibility' declaration, GSE65696, is incorrect. The correct number is GSE62696.

\section{Author details}

'Laboratory Animal Research Center, Zhejiang Chinese Medical University, Hangzhou 310053, China. ${ }^{2}$ Department of Cancer Genetics, Roswell Park Cancer Institute, Elm and Carlton Streets, Buffalo, NY 14263, USA. ${ }^{3}$ College of Animal Science, Nanjing Agricultural University, Nanjing 310058, China.

Published online: 16 January 2020

\section{Reference}

1. Cai, et al. Transcriptomic analysis of hepatic responses to testosterone deficiency in miniature pigs fed a high-cholesterol diet. BMC Genomics. 2015;16:59. https://doi.org/10.1186/s12864-015-1283-0.

Full list of author information is available at the end of the article 